\title{
Novel method to compute drag force and heat transfers for motions around spheres
}

\section{Méthode originale pour le calcul de la force de traînée et du transfert de chaleur pour des écoulements autour des sphères}

\author{
Mohamed-Amine Chadil ${ }^{1,2}$, Stéphane Vincent ${ }^{3}$, Jean-Luc Estivalezes ${ }^{1,4}$ \\ ${ }^{1}$ Institut de Mécanique des Fluides de Toulouse, IMFT, Université de Toulouse, CNRS - Toulouse, France \\ ${ }^{2}$ Institut de Mécanique et Ingénierie (I2M), Université de Bordeaux, France \\ ${ }^{3}$ Laboratoire MSME, UMR CNRS 8208, Université Paris-Est Marne-La-Vallée, France \\ ${ }^{4}$ The French Aerospace Lab, ONERA, Toulouse, France \\ Email : amine.chadil@imft.com, stephane.vincent@u-pem.fr, jean-luc.estivalezes@onera.fr
}

RÉSUMÉ. Une méthode de pénalisation visqueuse est utilisée pour simuler l'interaction des sphères et le fluide porteur en utilisant des simulations numériques directes avec particules résolues. Une méthode originale a été développée et validée afin d'extraire de ces simulations les forces hydrodynamiques et les transferts de chaleur sur des frontières immergées représentant les particules grâce aux extensions Aslam (2004). Cette méthode est une amélioration d'un travail précédent basé sur les extrapolations de Lagrange (chadil 2018). Des comparaisons entre ces deux approches sont conduites pour différents écoulements incompressibles, tels que l'écoulement autour d'une particule isolée à différents nombres de Reynolds et l'écoulement autour des sphères compactées dans des arrangements mono- et bi-dispersés cubiques à faces centrées.

ABSTRACT. A viscous penalty method is used to simulate the interaction between spheres and flows with ParticleResolved Direct Numerical Simulations. An original method has been developed and validated in order to extract from these simulations the hydrodynamic forces and heat transfers on immersed boundaries representing the particles thanks to Aslam extensions [3]. This method is an improvement of a previous work based on Lagrange extrapolations [6, 7]. Comparisons between these two approaches are considered on various incompressible motions such as the flow around an isolated particle at various Reynolds numbers and flows across packed spheres under Faced-Centered Cubic monoand bi-disperse arrangements.

MOTS-CLÉS. Particule résolue, coefficient de traînée, nombre de Nusselt, extension d'Aslam.

KEYWORDS. Particle-Resolved, Drag Coefficient, Nusselt number, Aslam extension.

\section{Introduction}

Fluid/Solid flows are widely encountered in nature as well as in various industrial processes. Among the wide variety of applied problems, we can cite volcanic eruptions [4, 11], oil refining, blast furnaces or chemical looping combustion [1, 23, 26], fluid catalytic cracking reactors [2], gas phase polymerization reactors [16] and fluidized beds [27, 40]. Their modeling and simulation at the application scale are generally based on statistical approaches where macroscopic Eulerian-Eulerian or mesoscopic EulerianLagrangian models (the average interphase transfer of momentum and energy between the fluid and the particles) are needed to be modeled.

Numerous well-known drag force laws are classically used in large scale models. The most popular are Schiller and Naumann [31] correlation for a single sphere whereas for fixed and fluidized beds of spheres, the correlations proposed by Ergun [14] and Wen and Yu [42] are often utilized. Heat transfers have also extensively been modeled for a single sphere using Ranz and Marshall [29] correlation, as well as for random arrangements of spheres with Gunn [17] law, to name the most famous of them.

Particle-Resolved Direct Numerical Simulation (PR-DNS) has been the privileged tool of many researchers in order to model unclosed terms in macroscopic statistical equations. Many numerical approaches 
have been developed to perform PR-DNS of gas-solid flows. These can be classified in two types of approaches. The first one rely on body-fitted mesh to impose boundary conditions at particle surface. Many codes use this technique, as for instance AVBP code [32] used by Massol [25] to conduct his work. The second type of approaches is called Fictitious domain methods. They employ a fixed Cartesian grid in the whole domain, where each phase is located thanks to phase or color function $C=1$ in the solid phase and $C=0$ in the fluid phase. It is utilized in Lattice Boltzmann approach [5, 18, 19, 21, 22]. Another class of very popular fictitious domain method for handling finite-size particle on fixed meshes is the Immersed Boundary Method (IBM) [12, 35, 38, 39, 41, 44]. The approach used in this work is the Viscous Penalty Method $[9,40]$. We have recalled here the most used methods in the literature.

The treatment of the fluid/solid interface in Navier-Stokes equations results in contaminated solution fields that do not correspond to unmodified Navier-Stokes equations. Tenneti et al. [36] proposed what they called the PUReIBM method where the immersed boundary (IB) forcing is restricted to the Eulerian grid points that lie in the solid phase, ensuring that the flow solution in the fluid phase is uncontaminated by the IB forcing. This allows them to easily compute drag forces [36] and heat transfers [33, 37]. In the case of VPM that we consider, we proposed, in a previous work [7], a Lagrange extrapolation for the drag force computation in order to reach the uncontaminated fluid area far from the particle/fluid interface ensuring in that way accurate results for drag coefficients. This method was used also in the heat transfer computation [6], and extended to ellipsoidal particles [8].

The purpose of this work is to propose an original alternative to Lagrange extrapolation in the hydrodynamic force and heat transfer computation in order to avoid contaminated solution of the Navier-Stokes equations near the solid-fluid interface. This original method is based on Aslam extension [3] to extrapolate velocity, pressure and temperature from the uncontaminated fluid area to the interface.

This paper is structured as follows. In section 1, the model and numerical methods used in the VPM are briefly recalled and a description as well as a validation of Aslam extension are detailed. Thereafter, section 2 is devoted to setting up Aslam extension numerical parameters and estimating drag and Nusselt coefficient for uniform flows around an isolated sphere and comparing them to those given by Lagrange extrapolation in order to assess the improvements made by Aslam extension. The same study is presented in section 3 for flows through fixed arrangements of mono- and bi-dispersed spheres. Conclusions are finally drawn in section 4 .

\section{Numerical Methodology}

\subsection{Conservation equations}

As explained by [9, 40], the motion equations describing incompressible two-phase flows that involve a carrier fluid and a solid particle phase are based on the one-fluid model. Their formulation is given by :

$$
\begin{array}{r}
\nabla \cdot \mathbf{u}=0 \\
\rho\left(\frac{\partial \mathbf{u}}{\partial t}+(\mathbf{u} \cdot \nabla) \mathbf{u}\right)=-\nabla p+\rho \mathbf{g}+\nabla \cdot\left[\mu\left(\nabla \mathbf{u}+\nabla^{t} \mathbf{u}\right)\right] \\
\rho C_{p}\left(\frac{\partial T}{\partial t}+\mathbf{u} \cdot \nabla T\right)=\nabla \cdot\left(k_{f} \nabla T\right)
\end{array}
$$


where $\mathbf{u}$ is the fluid or solid velocity, $p$ the pressure, $T$ the temperature, $t$ the time, $\mathbf{g}$ the gravity vector, $\rho, \mu, C_{p}$ and $k_{f}$ respectively the density, the viscosity, the specific heat and the thermal conductivity of the equivalent fluid depending on a local solid fraction $C$. This phase function is obtained by projecting the shape of the particles on the Eulerian fixed mesh. The advection equation on $C$ is not reported in the one-fluid model as only fixed particles are considered in the present work.

\subsection{Fictitious domain approach and viscous penalty method}

In the present work, fixed staggered Cartesian grids are used to discretized both fluid and solid media. The particles are viewed as fictitious domains sliding with their own Lagrangian surface mesh onto the fixed mesh used to solve the conservation equations (1-3). The details of the viscous penalty method and fictitious domain approach considered here are provided in $[7,28,40]$.

In a first approach, only motionless particles are considered in this work. On a numerical point of view, they are fixed by imposing the velocity of the Eulerian cells near their centroids to zero, and the viscous penalty method propagates the zero velocity in the whole solid medium. The particle temperatures will be considered constant over time, they are imposed at a fixed value in the whole solid medium also thanks to a penalty method used in the energy equation (3) [20].

\subsection{Drag force and heat flux computation using Aslam extension}

As mentioned in the introduction, the aim of this work is to propose an original method to compute the hydrodynamic force $\mathbf{F}$ and the heat flux $Q_{p}$ of a particulate flow from the velocity, pressure and temperature fields extracted from PR-DNS simulations. They are given by :

$$
\mathbf{F}=\oint_{S} \overline{\bar{\sigma}} \cdot \mathbf{n} d S \quad \text { and } \quad Q_{p}=\oint_{S}-K_{f} \nabla T \cdot \mathbf{n} d S
$$

The computation of $\mathbf{F}$ and $Q_{p}$ consists in discretizing the particle surface $S$ on a set of $N$ elements called Lagrangian mesh (see figure 1), such that :

$$
\mathbf{F} \approx \sum_{l}^{N} \overline{\bar{\sigma}}_{l} \cdot \mathbf{n}_{l} d S_{l} \quad \text { and } \quad Q_{p} \approx \sum_{l}^{N}-k_{f}(\nabla T)_{l} \cdot \mathbf{n}_{l} \Delta S_{l}
$$

where $k_{f}$ is the thermal conductivity, $\overline{\bar{\sigma}}_{l}$ and $(\nabla T)_{l}$ are the fluid stress tensor and the temperature gradient located at the $l^{\text {th }}$ element center $C_{l}, \mathbf{n}_{l}$ is the ongoing normal to the $l^{\text {th }}$ element and $\Delta S_{l}$ its area.

When $\mathbf{n}_{l}$ and $\Delta S_{l}$ are easily deduced from the nodes coordinates of the $l^{\text {th }}$ element, getting the stress tensor $\overline{\bar{\sigma}}_{l}$ and the temperature gradient $(\nabla T)_{l}$ at the $l^{\text {th }}$ element center $C_{l}$ is not straightforward when viscous penalty method is used to simulate fluid-solid flows. Indeed, velocity, pressure and temperature values, solution of Eqs (1-3), are inaccurate in the Eulerian cells cut by the interface [6, 7]. To address this numerical issue, a Lagrange extrapolation coupled to a Taylor interpolation of third order was proposed to extrapolate the stress tensor [7] and the temperature gradient [6] from the fluid area far from the interface to the Lagrangian mesh (see figure 1). This approach was fully detailed and validated in [6, 7]. These validations showed the efficiency of the method in the computation of the drag force and heat flux. 


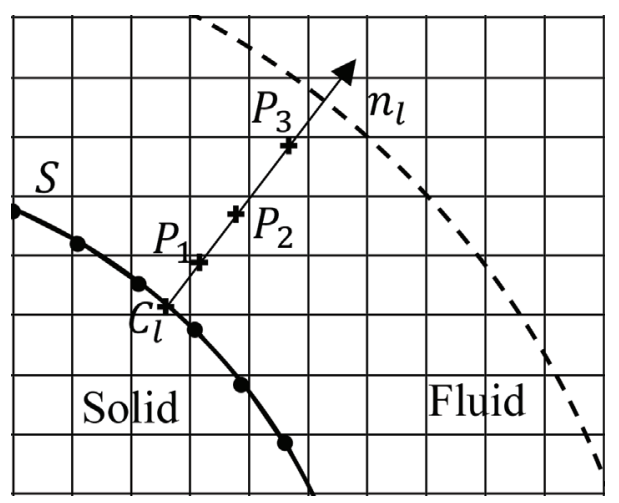

Figure 1. Details of a 2D discretization of the particle surface $S$ and extrapolation points $P_{i}$ of the third order Lagrange method used in drag force and heat flux computation.

Indeed the drag coefficient and the Nusselt number, both given bellow, were found in good agreement with many reference results of the literature.

The force and heat transfer coefficients at the particle surface are defined as follows :

- Drag coefficient :

$$
C_{d}=\frac{|\mathbf{F}|}{\frac{1}{2} \rho \tilde{U}^{2} A_{p}}
$$

where $\tilde{U}=\left|U_{\infty} \mathbf{e}_{x}-\mathbf{U}_{p}\right|$ is the relative velocity between the particle and the fluid velocity at infinity, $U_{\infty} \mathbf{e}_{x}$ is the fluid velocity in the mean flow direction far from the particle, $\mathbf{U}_{p}$ is the particle velocity, $\nu$ is the fluid kinematic viscosity and $A_{p}=\frac{\pi}{4} d^{2}$ the cross-sectional area of the particle.

- Nusselt number :

$$
N u=\frac{Q_{p} d}{k_{f}\left(T_{s}-T_{f}\right) S_{p}}
$$

where $Q_{p}$ is the heat flux, $k_{f}$ the thermal conductivity and $S_{p}=\pi d^{2}$ the area of the sphere. The temperatures of the particle and the fluid are respectively $T_{s}$ and $T_{f}$.

By using the Lagrange extrapolation coupled to the Taylor interpolation of $3^{\text {rd }}$ order, some oscillations were observed in the distribution of both the pressure coefficient given in the spherical system (see the figure 2) by :

$$
C_{p}(\theta)=\frac{p(\theta)}{\frac{1}{2} \rho U_{\infty}^{2}}
$$

and the local Nusselt number

$$
N u_{l o c}(\theta)=\frac{-\nabla T \cdot \mathbf{n} d}{\left(T_{s}-T_{f}\right)}
$$

especially at low Reynolds number $[6,7]$. These oscillations could be an explanation of some differences observed between our results and literature references, even if these oscillations were observed only for a few cases among the multitude conducted in $[6,7]$.

Another way to extrapolate the velocity, pressure and temperature from the fluid area, where they are not contaminated by the viscous penalty, to the region containing the interface, is proposed here to improve the accuracy of the current hydrodynamic force and the heat flux computations results. It is based on Aslam extensions [3]. 


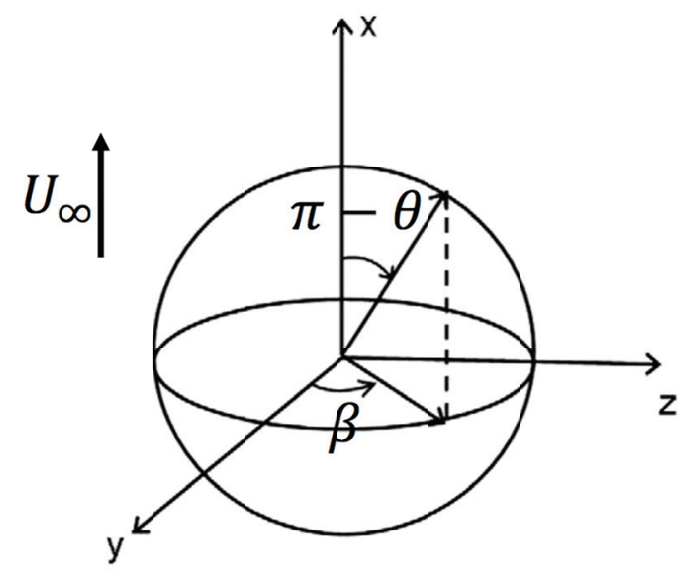

Figure 2. Spherical coordinate system around a particle. The flow direction is represented by the undisturbed velocity $U_{\infty}$.

\section{Description of Aslam extension}

Lets consider a sphere of diameter $d$ circumscribed by a surface $S$, and a domain $\Omega$ divided in three sub-domains as illustrated in Fig. 3 :

- $\Omega_{1}$ the area inside the sphere of radius $\left(\frac{d}{2}-\Delta x\right)$, where $\Delta x$ is the Eulerian cell mesh size.

- $\Omega_{2}$ the area outside the sphere of radius $\left(\frac{d}{2}+\delta\right)$. This is the region where the velocity, pressure and temperature are not contaminated by the viscous penalty. $\delta$ is then the distance between $\Omega_{2}$ and the interface.

- a band $\mathcal{B}$ bounded by the surfaces of the two sphere of radius $\left(\frac{d}{2}-\Delta x\right)$ and $\left(\frac{d}{2}+\delta\right)$ respectively. It contains the interface $S$. It is represented as the grey area in Fig. 3.

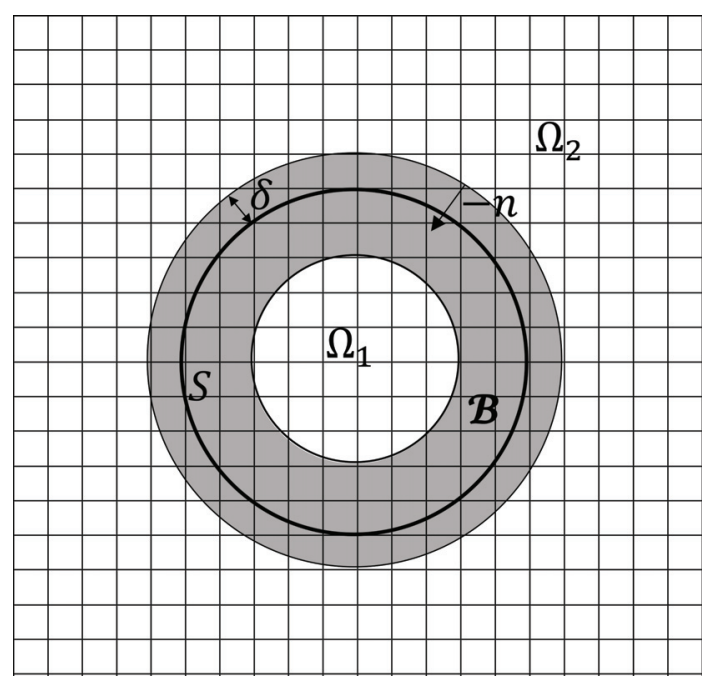

Figure 3. Details of the domain subdivision for Aslam extension [3]

Consider also a function $g$ defined in $\Omega_{2}$. For example, $g$ is a velocity component or the pressure used to compute the hydrodynamic force or either the temperature utilized to estimate the heat flux coefficient at the particle surface. Applied to these quantities, the Aslam extension [3] consists then in extrapolating $g$ from $\Omega_{2}$ to $\mathcal{B}$ by solving a series of advection equations given by [9]. 
Assuming a signed distance level set function $\psi$ is available on $\mathcal{B}(\psi>0$ inside the particle), a normal field to $S$ inside the band can be defined as :

$$
\mathbf{n}=\frac{\nabla \psi}{\|\nabla \psi\|}
$$

In our approach and for a particle of any form, $\psi$ is computed by a Ray-casting Method [30] based on the use of the Lagrangian mesh describing the particle surface $S$. However, for a spherical particle of diameter $d$ and centred in $\mathbf{x}_{\mathbf{0}}, \mathbf{n}$ and $\psi$ are given by :

$$
\forall \boldsymbol{x} \in \mathcal{B} \quad \mathbf{n}=\frac{\boldsymbol{x}-\boldsymbol{x}_{\mathbf{0}}}{\left\|\boldsymbol{x}-\boldsymbol{x}_{\mathbf{0}}\right\|} \quad \psi=\frac{d}{2}-\left\|\boldsymbol{x}-\boldsymbol{x}_{\mathbf{0}}\right\|
$$

To perform a $m^{\text {th }}$ order Aslam extrapolation, the equations [9] are successively resolved for decreasing values of $k=m, m-1, \ldots, 1$ :

$$
\frac{\partial g_{k}}{\partial \sigma}+H \nabla g_{k} \cdot(-\mathbf{n})=g_{k+1}
$$

where $\sigma$ is a fictitious time, $H$ is a Heaviside function defined by :

$$
H(\boldsymbol{x})= \begin{cases}1 & \text { if } \boldsymbol{x} \in \mathcal{B} \\ 0 & \text { otherwise }\end{cases}
$$

and $g_{k}$ is the projection on the normal field of the $k^{t h}$ order differential of the function $g$ :

$$
\begin{aligned}
g_{1} & =g \\
g_{2} & =\nabla g_{1} \cdot(-\mathbf{n}) \\
g_{3} & =\nabla g_{2} \cdot(-\mathbf{n}) \\
& \vdots \\
g_{m} & =\nabla g_{m-1} \cdot(-\mathbf{n}) \\
g_{m+1} & =0
\end{aligned}
$$

these derivatives are computed in $\Omega_{2}$ using a second-order upwind finite difference scheme :

$$
\left(g_{k}\right)_{i}=\frac{3\left(g_{k-1}\right)_{i}-4\left(g_{k-1}\right)_{i-1}+\left(g_{k-1}\right)_{i-2}}{2 \operatorname{sgn}\left(\boldsymbol{n}_{i}\right) \Delta \boldsymbol{x}_{i}} \boldsymbol{n}_{i}
$$

Using an upwind scheme rather than a Central finite difference scheme as proposed in Aslam [3] allows the use of the same Heaviside function $H$ for all $m$ equations [9] resolved for the $m^{\text {th }}$ order Aslam extension, and not a specific Heaviside function $H_{k}$ for each equation as proposed by Aslam [3].

The equations [9] are resolved until steady state is reached, using a first-order upwind finite difference scheme for the time derivative and a second-order TVD "SuperBee" scheme [34] for the space derivative. It is worth noting that when the steady state is reached in the $\mathcal{B}$ band, $g$ verifies in $\mathcal{B}$ :

$$
\begin{aligned}
\nabla g_{m} \cdot(-\mathbf{n}) & =0 \\
\nabla g_{m-1} \cdot(-\mathbf{n}) & =g_{m} \\
& \vdots \\
\nabla g_{2} \cdot(-\mathbf{n}) & =g_{3} \\
\nabla g \cdot(-\mathbf{n}) & =g_{2}
\end{aligned}
$$


Once the velocity and pressure fields for hydrodynamic force computation and temperature for heat flux computation, are extrapolated in the band $\mathcal{B}$ using the $m^{\text {th }}$ order Aslam extension, the stress tensor and the temperature gradient are computed in $\mathcal{B}$ and then interpolated onto the Lagrangian mesh using a $m^{\text {th }}$ order Taylor interpolation which was fully detailed in [7]. This interpolation is formulated as :

$$
g\left(\boldsymbol{C}_{l}\right)=\sum_{|\alpha|=0}^{m-1} \frac{1}{\alpha !} \frac{\partial^{\alpha} g}{\partial x^{\alpha}}(\boldsymbol{E})\left(\boldsymbol{C}_{l}-\boldsymbol{E}\right)^{\alpha}+\mathrm{O}\left(\left\|\boldsymbol{C}_{l}-\boldsymbol{E}\right\|^{m}\right)
$$

where $\alpha=\left(\alpha_{1}, \alpha_{2}, \alpha_{3}\right) \in \mathbb{N}^{3}$ is the sum multi-index and $\boldsymbol{E}$ denotes the nearest Eulerian point to the point $\boldsymbol{C}_{l}$, as illustrated in Fig. 4 for a third order Taylor interpolation.

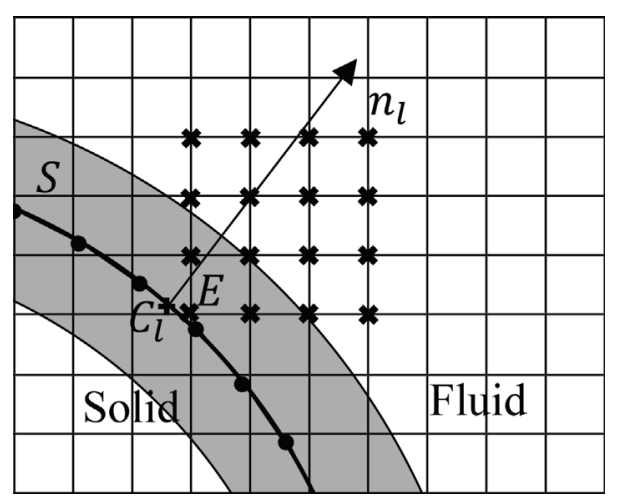

Figure 4. Details of Taylor interpolation points for drag force/heat flux computation at point $\mathbf{C}_{1}$ of the surface Lagrangian mesh. E is the nearest fluid point to the particle/fluid interface. The crosses represent the discrete compact support of the fluid points required for a $3^{r d}$ order Taylor interpolation.

\section{Validation of Aslam extension}

To validate these extension we used the same example as Aslam [3] i.e. a $[-\pi, \pi]^{2} 2 \mathrm{D}$ domain with a particle located at the center of the domain and a function $g$ to be extrapolated given by :

$$
g(\boldsymbol{x})= \begin{cases}\cos \left(\boldsymbol{x}_{1}\right) \sin \left(\boldsymbol{x}_{2}\right) & \text { if } \psi(\boldsymbol{x})>0 \quad \text { (i.e. inside the particle) } \\ 0 & \text { otherwise }\end{cases}
$$

In this example the function $g$ is defined inside the particle i.e. in $\Omega_{1}$. It has to be extrapolated in the band $\mathcal{B}$ as illustrated in Figure 5.

This example was extended to non-spherical particles, with an ellipse and a square. Figure 6 shows the contours of $g$ inside $(a)$ a circle, $(b)$ an ellipse and $(c)$ a square which will be extrapolated in $\mathcal{B}$ i.e. the delimited white zone outside the particle, using equations [9].

The function $g$ is extrapolated using Aslam extension, i.e. resolving equations [9], for four different orders :

— Constant Aslam extension $m=1$ : it consists in resolving equation $\frac{\partial g}{\partial \sigma}+H \nabla g \cdot \mathbf{n}=0$ in $\mathcal{B}$ until $g$ reaches a steady state i.e. $\frac{\partial g}{\partial \sigma}=0$, i.e. $\frac{\partial g}{\partial \mathbf{n}}=\nabla g \cdot \mathbf{n}=0$, which means that extrapolated $g$ is constant on the normal direction to the particle surface in the extrapolation region $\mathcal{B}$ as illustrated in Figure 7. 


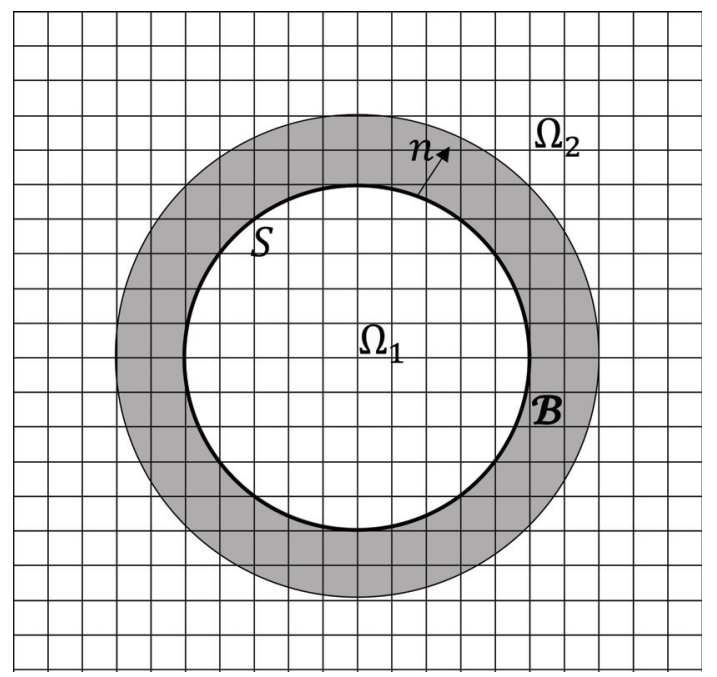

Figure 5. Details of the Aslam extension validation example.

(a)

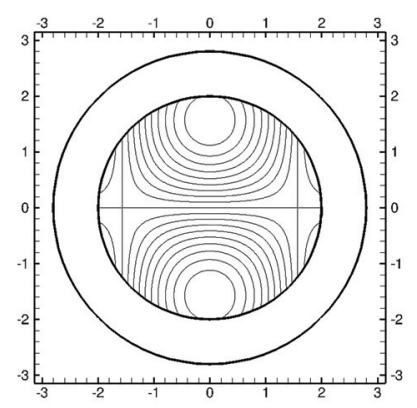

(b)

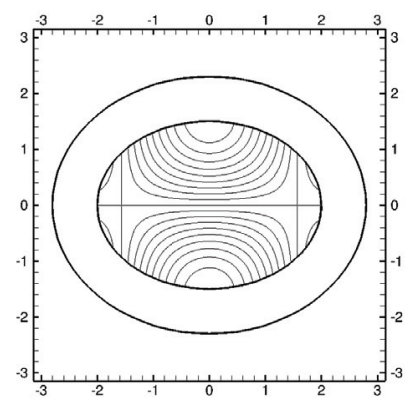

(c)

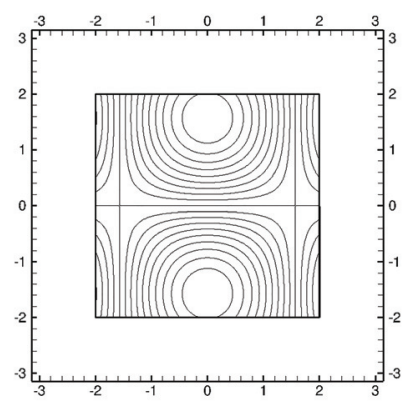

Figure 6. Contour (with a 0.2 increment) of the initials conditions of $g$ for Aslam extension from : $(a)$ a circle, $(b)$ an ellipse and $(c)$ a square.

(a)

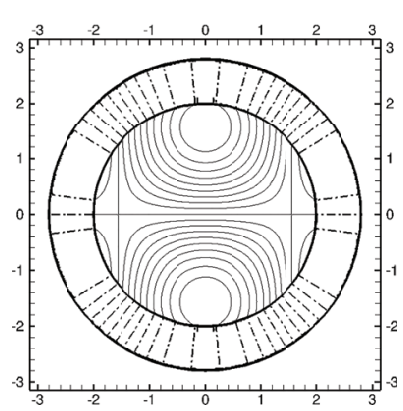

(b)

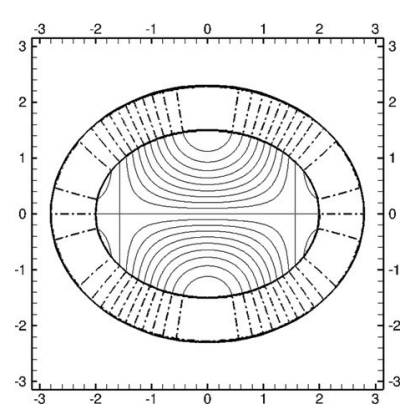

(c)

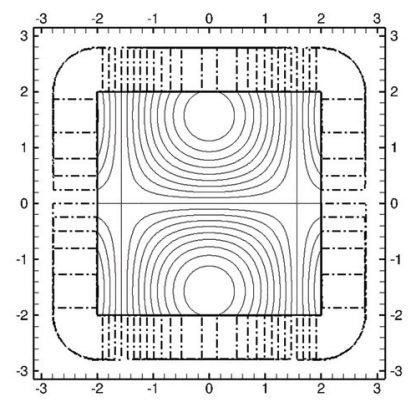

Figure 7. Contour (with a 0.2 increment) of the function $g$ extrapolated using Constant Aslam extension $(m=1)$ from : $(a)$ a circle, $(b)$ an ellipse and $(c)$ a square. 
- Linear Aslam extension $m=2:$ it consists in extrapolating the first normal derivative $g_{2}=\frac{\partial g}{\partial \mathbf{n}}$ from $\Omega_{1}$, where it is computed from $g$ using [10], to the band $\mathcal{B}$ using Constant Aslam extension. Then $g$ is extrapolated from $\Omega_{1}$ to $\mathcal{B}$ by resolving equation $\frac{\partial g}{\partial \sigma}+H \nabla g \cdot \mathbf{n}=g_{2}$ until $g$ reaches a steady state. An illustration of such function is given in Figure 8 where one can observe that $g$ is no more constant on the normal direction to the particle surface in $\mathcal{B}\left(\nabla g \cdot \mathbf{n}=g_{2}\right)$.

(a)

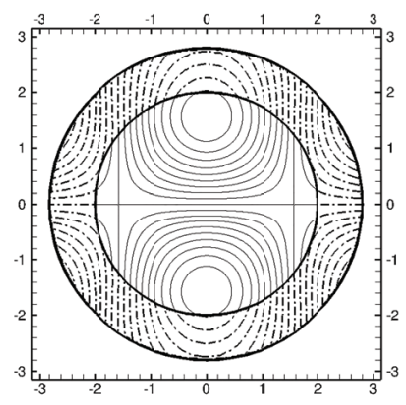

(b)

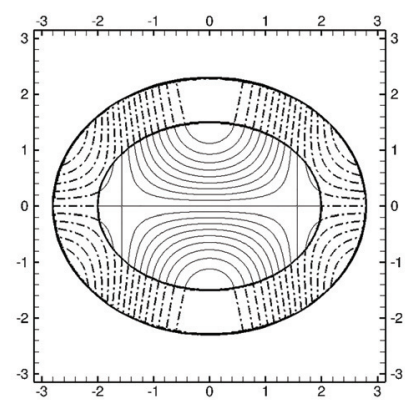

(c)

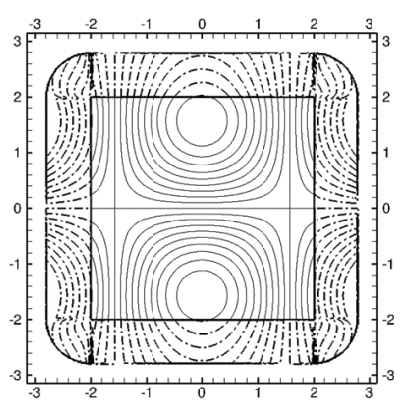

Figure 8. Contour (with a 0.2 increment) of the function $g$ extrapolated using Linear Aslam extension $(m=2)$ from : $(a)$ a circle, $(b)$ an ellipse and $(c)$ a square.

- Quadratic Aslam extension $m=3$ : it consists in extrapolating the second normal derivative $g_{3}=\frac{\partial g_{2}}{\partial \mathbf{n}}=\frac{\partial^{2} g}{\partial \mathbf{n}^{2}}$ from $\Omega_{1}$,where it is computed from $g_{2}$ using [10], to the band $\mathcal{B}$ using Constant Aslam extension. Then, $g_{2}$ is extrapolated using Linear Aslam extension with $g_{3}$ as second member of the extrapolation equation. And finally $g$ is extrapolated by resolving in $\mathcal{B} \frac{\partial g}{\partial \sigma}+H \nabla g \cdot \mathbf{n}=g_{2}$ until $g$ reaches a steady state. In Figure 9, the extrapolated part of $g$ seems to converge toward the initial function $g$. Note that the incoherent values of the extrapolated $g$ in the corner region for the square case are the consequence of the fact that the normal is unknown in this region.

(a)

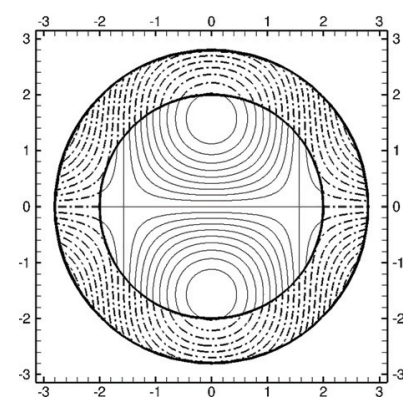

(b)

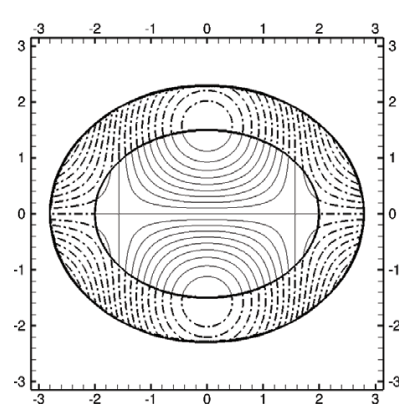

(c)

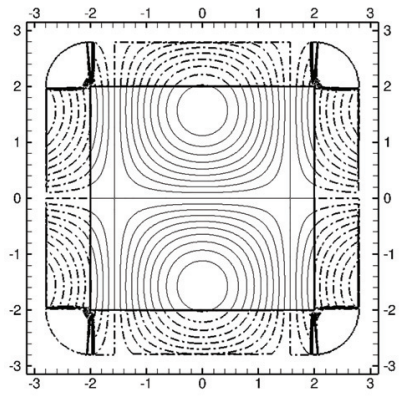

Figure 9. Contour (with a 0.2 increment) of the function $g$ extrapolated using Quadratic Aslam extension $(m=3)$ from : $(a)$ a circle, $(b)$ an ellipse and $(c)$ a square.

- Cubic Aslam extension $m=4:$ it consists in extrapolating the third normal derivative $g_{4}=\frac{\partial g_{3}}{\partial \mathbf{n}}$ from $\Omega_{1}$, to the band $\mathcal{B}$ using Constant Aslam extension. In a second step, $g_{3}$ is obtained using Linear Aslam extension with $g_{4}$ as second member of the extrapolation equation. Then $g_{2}$ is extrapolated using Quadratic Aslam extension with $g_{3}$ as second member of the extrapolation equation. Finally $g$ is approximated by resolving $\frac{\partial g}{\partial \sigma}+H \nabla g \cdot \mathbf{n}=g_{2}$ until $g$ reaches a steady state. The results obtained with this extrapolation are illustrated in Figure 10. 
(a)

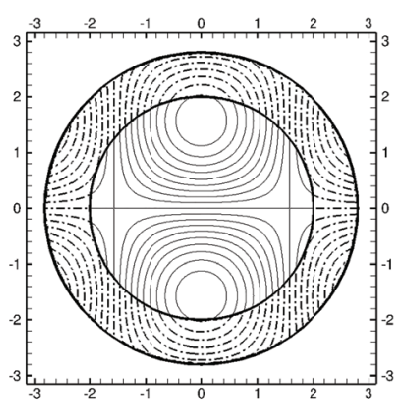

(b)

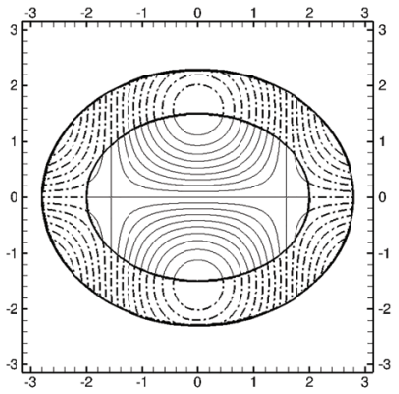

(c)

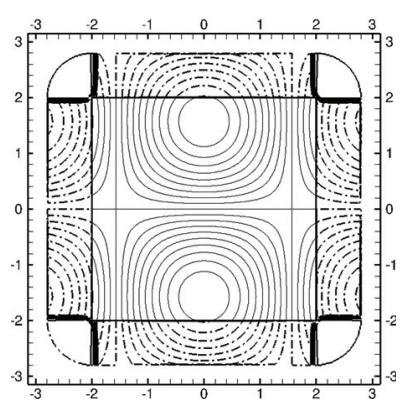

Figure 10. Contour (with a 0.2 increment) of the function $g$ extrapolated using Cubic Aslam extension $(m=4)$ from : $(a)$ a circle, $(b)$ an ellipse and $(c)$ a square.

A convergence study was conducted for these four Aslam extension orders using the relative error, in the band $\mathcal{B}$, between exact $g$ values and those obtained with extrapolation Aslam procedures. It can be observed in Figure 11 that these errors are always small (from 0.1 to $10^{-8} \%$ ), and that the expected orders are found for extensions of the circle and the ellipse shape. However, in the case of an extension from a square surface, the order could not be reached because of the inaccurate values in the region near the square corner, where the normal to the surface is not defined.

(a)

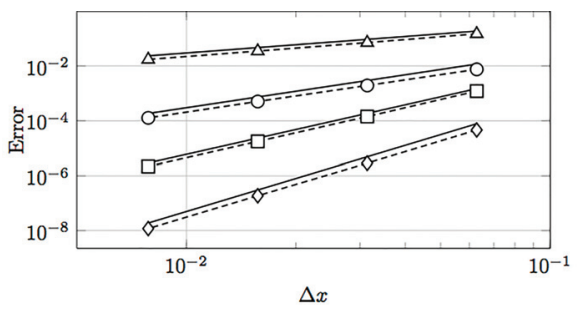

(b)

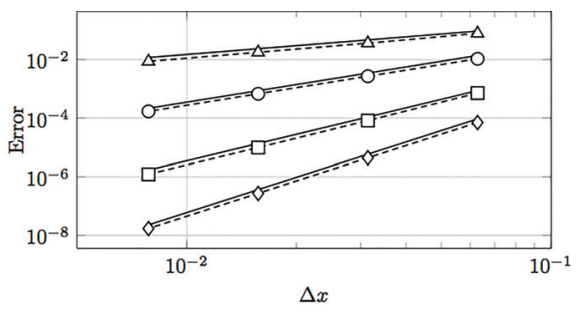

(c)

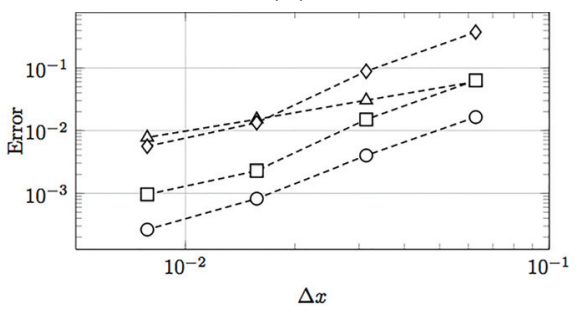

Figure 11. Convergence orders in 2 dimensions for Aslam extension from : $(a)$ a circle, $(b)$ an ellipse and $(c)$ a square. $(\triangle) m=1,(\bigcirc) m=2,(\square) m=3$ and $(\diamond) m=4$.

The exact same study was conducted for three dimensional cases. Indeed, a $[-\pi, \pi]^{3} 3 \mathrm{D}$ domain with a particle (sphere, ellipsoid, cube) located at the center of the domain and a function $g$ to be extrapolated are considered here to validate Aslam extension in 3D. Now, the function $g$ is defined by :

$$
g(\boldsymbol{x})= \begin{cases}\cos \left(\boldsymbol{x}_{1}\right) \sin \left(\boldsymbol{x}_{2}\right) \cos \left(\boldsymbol{x}_{3}\right) & \text { if } \psi(\boldsymbol{x})>0 \quad \text { (i.e. inside the particle) } \\ 0 & \text { otherwise }\end{cases}
$$

Figure 12 shows that, similarly as in the 2D cases, the expected extension orders are found for the sphere and the ellipsoid, even if the relative errors are two order of magnitude greater than in 2D cases. As for the 2D case, the non-definition of the normal to the cube in the vicinity of its corners lead to inaccurate values of the extrapolated function in the region near to these corners. This explains the errors behavior that makes the extension order computation impossible in this case. 
(a)

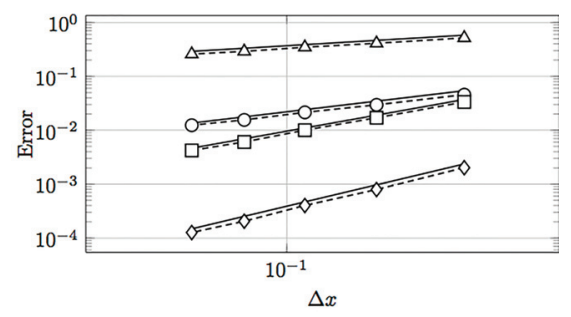

(b)

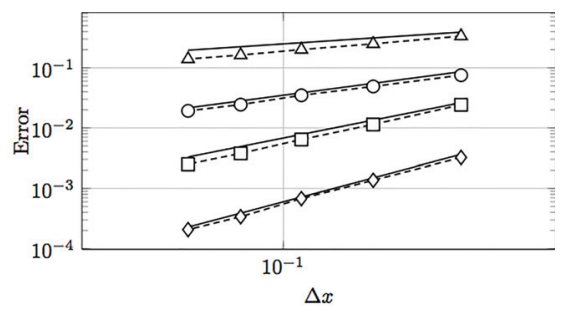

(c)

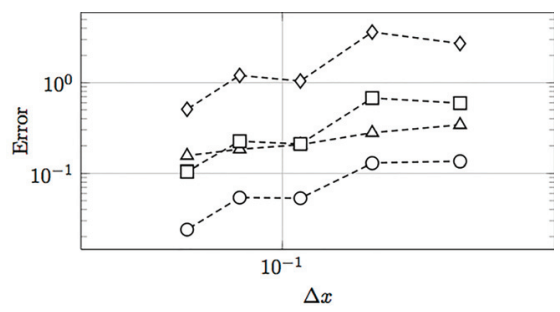

Figure 12. Convergence orders in 3 dimensions for Aslam extension from : $(a)$ a sphere, $(b)$ an ellipsoid and $(c)$ a cube. $(\triangle) m=1,(\bigcirc) m=2,(\square) m=3$ and $(\diamond) m=4$.

\section{Isolated stationary sphere past by a uniform flow}

The aim of this work is to assess how Aslam extension could improve the computation of the hydrodynamic force and the heat flux compared to the results obtained using Lagrange extrapolation. To do that, we conducted the same simulations carried out in $[6,7]$ to validate the force and heat transfer computation using Lagrange extrapolation. The first case considered here is a forced convection by uniform flow past a hot stationary sphere, illustrated in figure 13. All the details and description of this problem are reported in $[6,7]$.

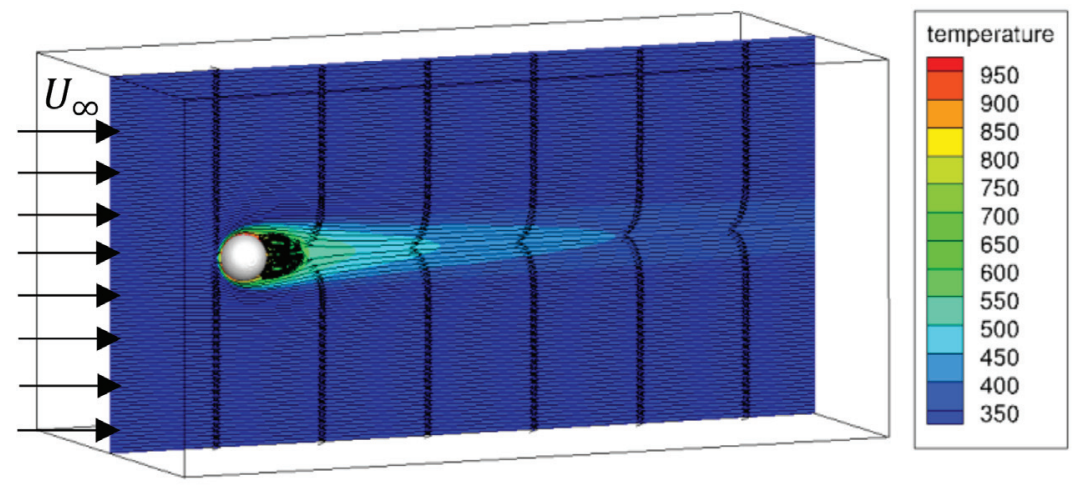

Figure 13. Streamlines and temperature field for a uniform flow past a sphere at $R e=100$.

\subsection{Drag force computation}

Following the steps of the study of the drag force computation using Lagrange extrapolation [7], we will first determine how far we have to go from the particle to reach uncontaminated velocity and pressure of the fluid to extrapolate them to the interface in order to get accurate values of drag force. The effect of Aslam extrapolation order will be studied at the same time on these values. Moreover, some perturbations were observed in the local pressure distribution on the particle surface in [7]. We will see how Aslam extension could prevent these oscillations. Finally, we will compare the drag coefficient values computed with Lagrange extrapolation [7] with those computed with Aslam extension. 
One of the main drag force computation parameter using Lagrange extrapolation, studied in [7], was the distance $(\delta)$ between the particle and the first point of extrapolation $\mathbf{P}_{1}$ (see Figure 1 ). Indeed, as reported in [7] and as illustrated in Figures (14 a) at Stokes regime and (14 c) at $R e=100$, the drag force values, computed using Lagrange extrapolation, are inaccurate for $\delta \leq 1$. This is due to contaminated values of velocity and pressure induced by the viscous penalty method [9] in the Eulerian cells cut by the interface. The study of the effect of this distance $\delta$ (illustrated in Figure 3 ) is conducted for drag force computation using Aslam extension as well. The Figures (14 b) (14 d) show that, in order to get an accurate drag force using Aslam extensions, a third order is required. Therefore, for the rest of this work, a third order Aslam extension coupled with a third order Taylor interpolation will be used in the drag force computation. One can also observe in these figures that the distance to go far from the interface is reduced to $\delta=0.5$ with Aslam approach. One possible explanation of this improvement is that, it is actually the pressure and the velocity that are extrapolated by Aslam extension in the drag force computation rather than the stress tensor components, i.e. the gradients of these variables, as it is the case when the Lagrange extrapolation is used. Indeed, the stress tensor is computed using centered difference schemes which utilizes contaminated values of velocity and pressure for $\delta \leq 1$ (and even solid values for $\delta=0$ ). On the other hand, extrapolating directly the velocity components and the pressure using Aslam extension and then computing the stress tensor from these extrapolated values reduces the distance to which how far we have to move away from the particle to reach accurate drag force values.

(a)

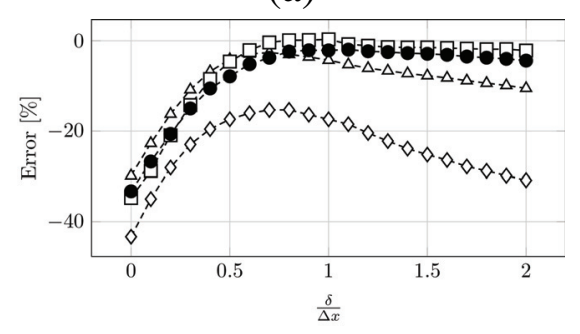

(c)

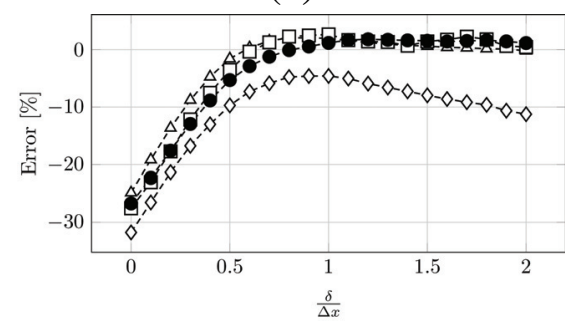

(b)

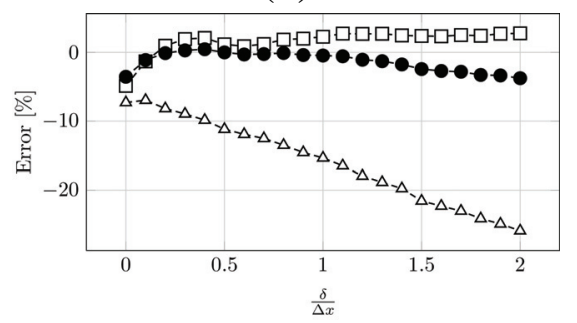

(d)

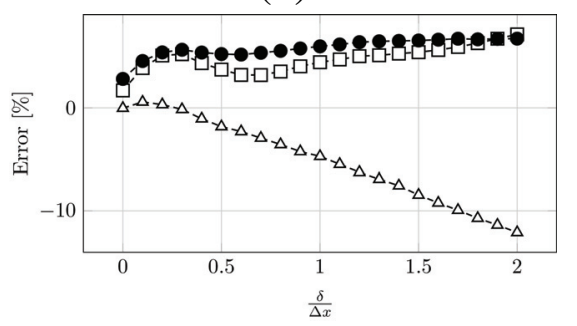

Figure 14. Drag force relative error $(\%)$ for the uniform flow past a sphere in Stokes regime $(a)$ for Lagrange extrapolation and $(b)$ for Aslam extrapolation and at $R e=100$ for $(c)$ Lagrange extrapolation and $(d)$ Aslam extrapolation. Different extrapolation orders are considered : $(\diamond)$ first, $(\triangle)$ second, $(\bullet)$ third, and ( $\square$ ) fourth order. The distance between the first Eulerian point used to extrapolate forces and the particle surface is $\delta$. 
The analysis of local pressure profiles in [7] was used to better understand the behavior of the pressure on the sphere depending on the Reynolds number. The local pressure coefficients are defined by equation [7] in a spherical coordinate system (see figure 2).

The pressure coefficient distribution according to $\theta$ was compared in [7] to some available body fitted simulations results conducted by Magnaudet et al. [24], Dennis and Walker [13], LeClair et al. [10] and Massol [25] at different Reynolds numbers. The results are presented in Figures (15 a), (15 c) and (15 e) for $R e=1,10,100$ respectively.

(a)

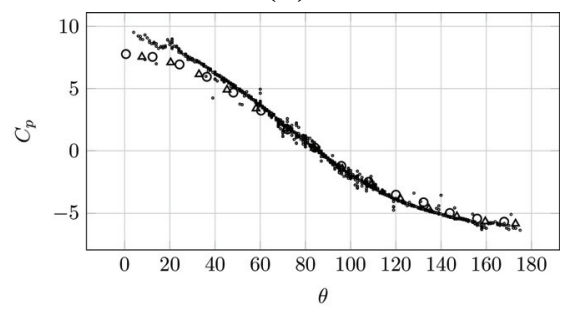

(c)

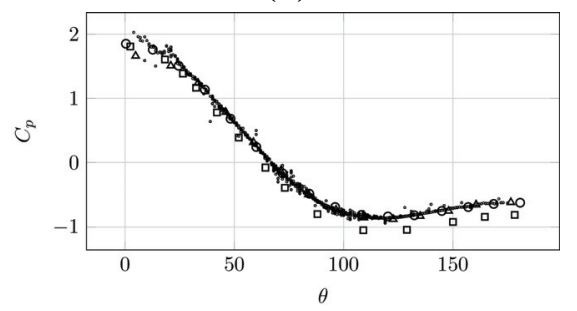

(e)

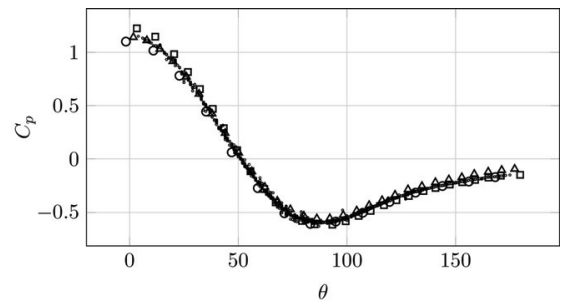

(b)

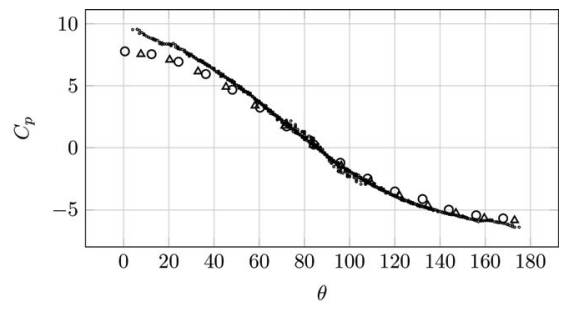

(d)

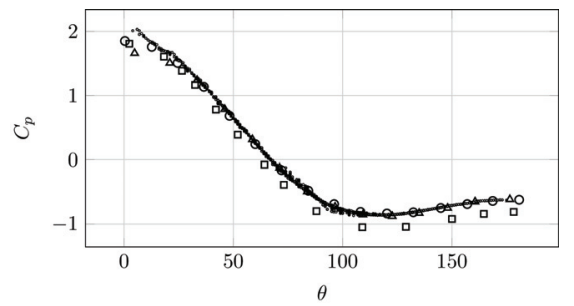

(f)

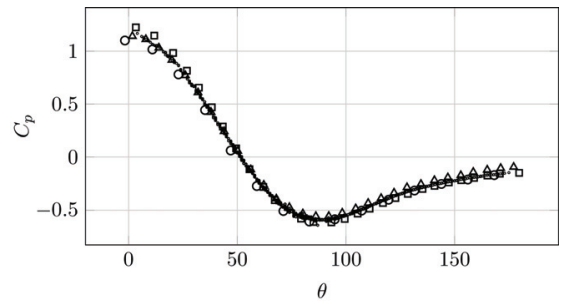

Figure 15. Pressure coefficient for a uniform flow past an isolated sphere at $R e=1$ : $(a)$ for Lagrange extrapolation, $(b)$ for Aslam extrapolation, $R e=10:(c)$ for Lagrange extrapolation, $(d)$ for Aslam extrapolation, $R e=100:(e)$ for Lagrange extrapolation, $(f)$ for Aslam extrapolation. $(\diamond)$ Dennis et al. [13], $(\triangle)$ Magnaudet et al. [24], (○) LeClair et al. [10], (口) Massol [25], and (.) present work.

It is well-known that, for this range of Reynolds number, the flow is symmetric with respect to its direction, as illustrated in Figure 16. Therefore, the pressure coefficient distribution must be the same for all the polar angle $(\beta)$ plans, translating by that the flow symmetry. This expected behavior was mostly observed when Lagrange extrapolation was used in the drag force computation [7] (see Figures (15 a), (15 c), (15 e)). However, some local pressure values diverge from the expected ones, especially for low Reynolds number $(R e=1,10)$ as illustrated in Figures $(15 \mathrm{a}),(15 \mathrm{c})$.

The use of Aslam extension in the drag force computation seems to reduce the pressure oscillations, which is a good point of the method, although only few pressure points exhibited values far from expected 


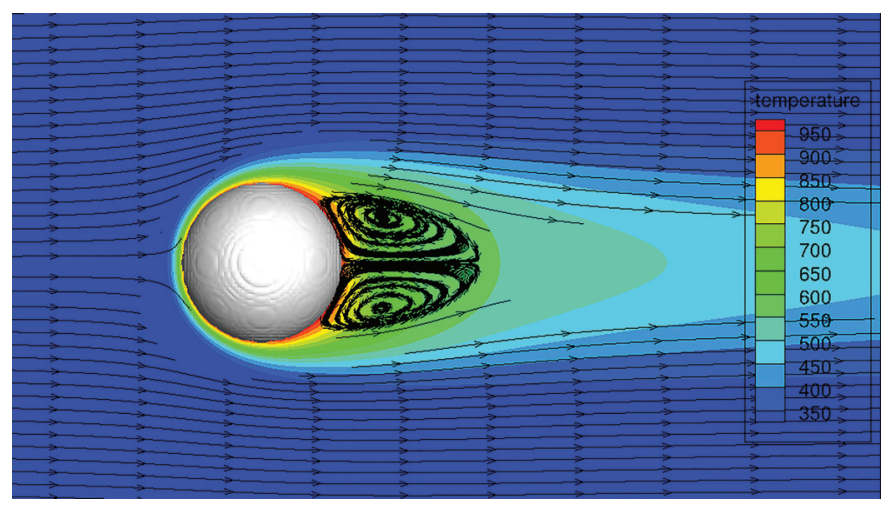

Figure 16. Streamlines and temperature field for a uniform flow past a sphere at $R e=100$.

ones when Lagrange extrapolation is used. Indeed, the same distribution of the pressure coefficient is observed for all $\beta$ plans in Figures (15 b), (15 d), (15 f).

\section{Drag coefficient}

The last step in the study of drag force computation, using a third order Aslam extension, is devoted to the drag coefficient, given by the Eq[5] and extracted from PR-DNS. It is compared to Schiller and Naumann [31] correlation for a wide range of Reynolds number $(R e=0.1-290)$. This comparison was conducted in [7] when the drag force was computed using Lagrange extrapolation.

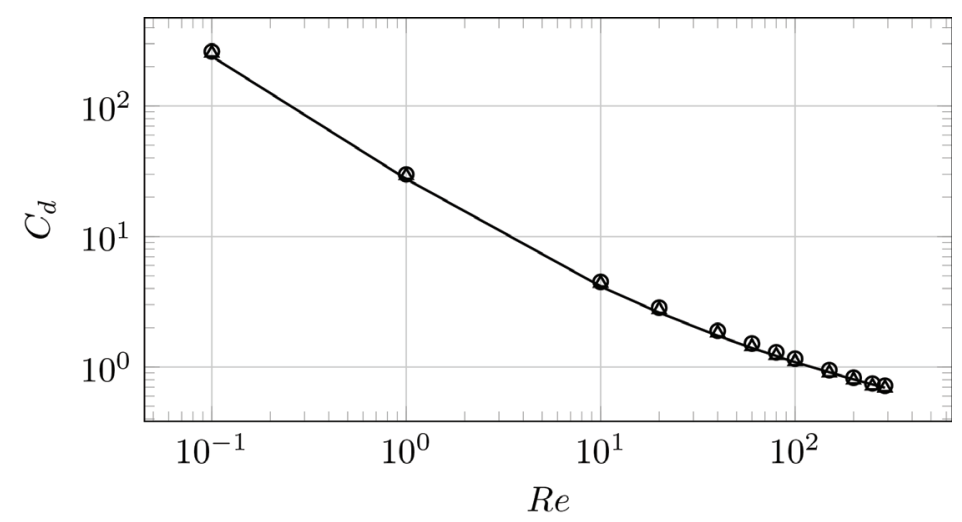

Figure 17. Drag coefficient for uniform flow past sphere at different Reynolds number : (-) Schiller and Naumann [31], $(\bigcirc)$ present work using Aslam extension and $(\triangle)$ using Lagrange extrapolation.

Figure 17 shows the good agreement of our results with Schiller and Naumann [31]. It also shows that, at least for this particular case, the drag force computed using Aslam extension is quite the same than the one computed using Lagrange extrapolation. Indeed, as it has been discussed earlier, Aslam extension improvement was the reduction of the distance $\delta$ to go away from the particle to get accurate drag force, and the correction of the few inaccurate values of the pressure on the particle surface. In the case of an isolated sphere the distance $\delta$ does not affect the outcome of the drag force as soon as the accurate value is found whatever the force calculation method used (Lagrange extrapolation or Aslam extension). On the contrary, in the case of arrangement of spheres which will be discussed in the next section, the distance $\delta$ will be an important parameter especially for high solid volume fraction. Moreover, the number of inaccurate pressure values in the pressure coefficient distribution is not important enough to really influence the total drag force in the present case of an isolated sphere. 


\subsection{Heat transfer computation}

The effect of choosing $\delta$ is now considered with respect to the heat transfer computation. This effect is estimated using the local Nusselt number computed using a third order Aslam extension [3] coupled to a third order Taylor interpolation, followed by a comparison of this local Nusselt number distribution to the one computed using Lagrange extrapolation [6]. Once the parameter $\delta$ is set, the global Nusselt number for the problem of a uniform flow past a hot sphere is compared to the correlations given by Ranz and Marshall [29], Feng and Michaelides [15] and Whitaker [43].

\section{Effect of the extrapolation distance $\delta$}

To study the $\delta$ effect, the local Nusselt number distribution was compared to the one provided by Massol [25] thanks to body-fitted PR-DNS results at $R e=100$. As previously explained for the pressure coefficients, this distribution is also expected to be symmetric with respect to the flow direction, as illustrated in Figure 16.

(a)

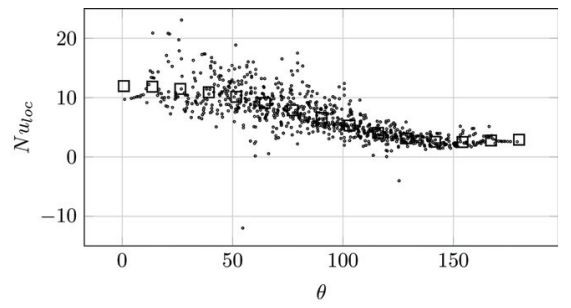

(c)

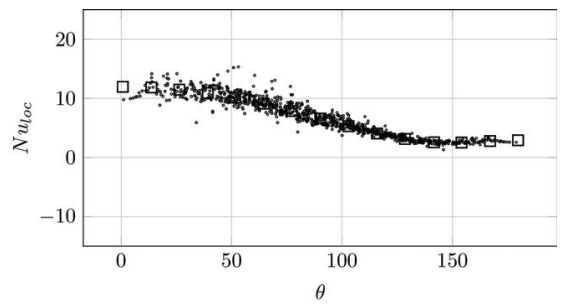

(b)

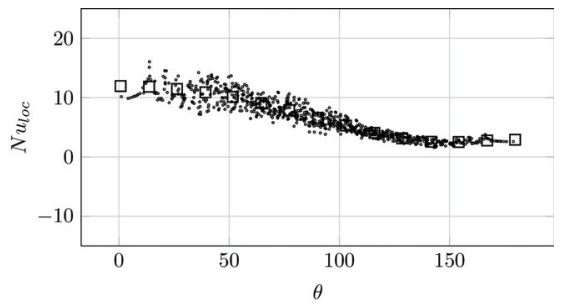

(d)

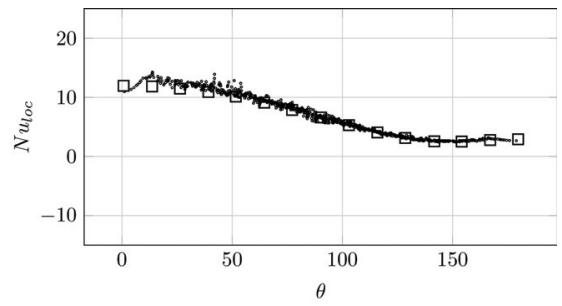

Figure 18. Local Nusselt coefficient for a uniform flow past an isolated sphere at $R e=100$, as function of the azimuthal angle $\theta$ at $\delta=0:(a)$ for Lagrange extrapolation, (b) for Aslam extrapolation, $\delta=1$ : $(c)$ for Lagrange extrapolation, $(d)$ for Aslam extrapolation. (.) present work, ( $\square$ ) Massol [25].

The local Nusselt number computed for $\delta=0$ does not reflect the flow symmetry neither for the one computed using Lagrange extrapolation ( see Figure 18 a) nor the one computed using Aslam extension (see Figure $18 \mathrm{~b}$ ), even if the latest improved sensibly the result with lower amplitude of Nusselt oscillations. Indeed, the error between our result $\left(N u_{l o c}\right)$ and Massol's $\left(N u_{\text {locMassol }}\right)$ given by $\frac{\sum_{i=1}^{N}\left|\left(N u_{l o c}\right)_{i}-\left(N u_{l o c M a s s o l}\right)_{i}\right|}{\sum_{i=1}^{N}\left|\left(N u_{\text {locMassol }}\right)_{i}\right|}$ (illustrated in Figure 19) is about $25 \%$ for Lagrange extrapolation and less than $15 \%$ for the Aslam extension. This error decreases as the distance $\delta$ increases until being lower than $10 \%$ for $\delta \geq 1$ in case of Lagrange extrapolation use, and about $5 \%$ in the case of Aslam extension use for $\delta=1$ as illustrated in Figure 19. Moreover, Figures ( $18 \mathrm{c}$ ) and ( $18 \mathrm{~d}$ ) show the distribution of the local Nusselt number for $\delta=1$ with respectively Lagrange and Aslam extrapolations and they show also that Aslam extension improves the Heat transfer computation with an almost symmetrical distribution of 
the local Nusselt number for $\delta=1$. Therefore, the extrapolation distance will always be $\delta=1$ in the rest of the present work.

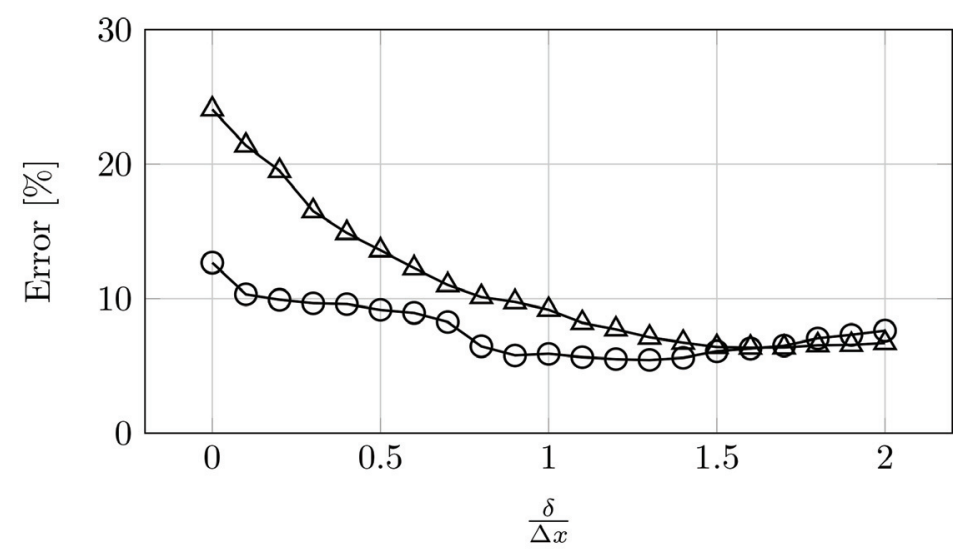

Figure 19. Local Nusselt number relative error compared to Massol's result [25] as a function of the extrapolation distance $\delta:(\bigcirc)$ using Aslam extension and $(\triangle)$ using Lagrange extrapolation.

\section{Nusselt number}

After setting up the numerical parameter for the heat transfer computation, the Nusselt number obtained when considering a uniform flow past a hot fixed sphere is computed using Aslam extension and compared to the values computed using Lagrange extrapolation [6] and to some of the existing correlations of the literature.

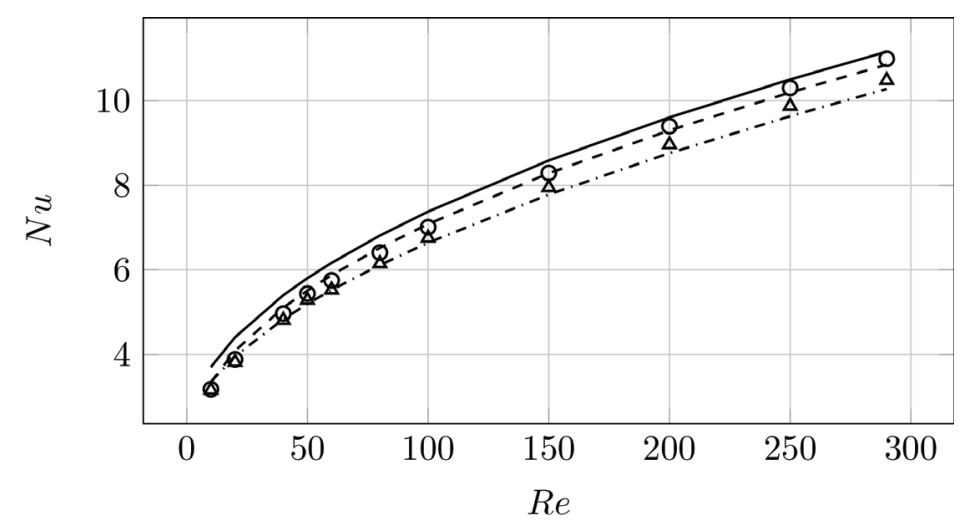

Figure 20. Nusselt coefficient for the uniform flow past a hot sphere at various Reynolds numbers : (-) Ranz and Marshall [29], (- -) Feng and Michaelides [15], (-...) Whitaker et al.. [43], (○) present work using Aslam extension and $(\triangle)$ using Lagrange extrapolation.

Figure 20 shows the good agreement of our results to correlations, with clear improvement when Aslam extension is used to compute the Nusselt number. Indeed, unlike the drag force computation and as it was discussed in the previous subsection, the Aslam extension has an effect on the obtained local Nusselt number values. This naturally improves the global Nusselt number that converges toward the well-known Ranz and Marshall correlation [29] (see Figure 20), which is expected to be the reference law used in the literature. 


\section{Face-Centered Cubic arrangement of stationary sphere past by a uniform flow}

As explained in subsection 2.1., Aslam extension does not improve the drag force computation of an isolated sphere. Indeed, Aslam extension reduced the distance $\delta$ to go far from the particle to reach accurate drag value, going from $\delta=1$ for Lagrange to $\delta=0.5$ for Aslam. Moreover, for an isolated sphere, this distance does not affect the computed value of the drag as long as an accurate value is reached whatever the extrapolation technique used (see Figure 14). However, it is obvious that for an assembly of spheres, the distance $\delta$ would affect the drag force computed on each sphere as the distance between the spheres reduces when the solid volume fraction increases. Two configuration of sphere assemblies , mono- and bi-dispersed arrangements of spheres, are studied in this section following in that the work done in $[6,7]$ when Lagrange extrapolation was used in the drag force and heat transfer computation in order to compare the two extrapolation method outcomes.

\subsection{Monodispersed Face-Centred Cubic periodic arrangement of spheres}

The first case considered here is a uniform flow past a Face-Centered Cubic (FCC) array of spheres. We wish to study whether or not Aslam extension improves the drag force and the heat transfer computations, when a sphere is surrounded by other particles, in comparison to those computed using Lagrange extrapolation. FCC arrangement of spheres consists in a cube where three spheres are placed on the faces centers, and one sphere is located on the vertices with periodic boundary conditions, as illustrated in the figure 21 . This case was fully detailed in $[6,7]$.

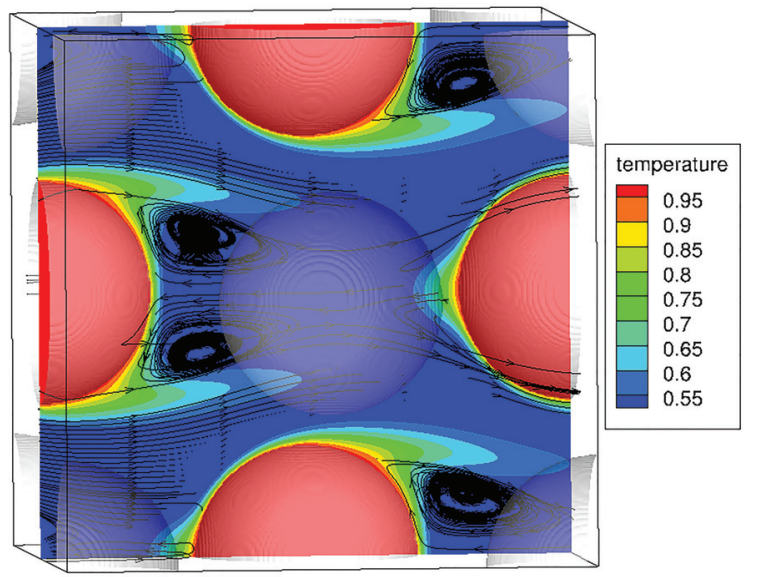

Figure 21. Streamlines and temperature field for a steady flow along the $\mathrm{x}$-axis of a Face-Centered Cubic array of spheres at $R e=300, \alpha_{d}=0.15$

\section{Drag force}

When the flow steady state is reached at the desired Reynolds number, as explained in [7], the mean non-dimensional drag force $F$ for all the particles is then deduced from the drag forces computed over each particle by :

$$
F=\frac{\left|\left\langle\mathbf{F}^{D}\right\rangle\right|}{3 \pi \mu d\left(1-\alpha_{d}\right)\left|\left\langle\mathbf{u}_{f}\right\rangle\right|}
$$


with $\left\langle\mathbf{F}^{D}\right\rangle=\frac{1}{N_{p}} \sum_{i}^{N_{p}} \mathbf{F}_{i}^{D}, \mathbf{F}_{i}^{D}$ being the drag force computed over the $i^{t h}$ particle.

The non-dimensional drag force $F$ normalized by the isolated sphere non-dimensional drag force (given by Schiller and Naumann [31]) $F_{s}=\frac{1+0.15 R e^{0.687}}{\left(1-\alpha_{d}\right)^{2}}$, noted as $\frac{F}{F_{s}}$, is compared to Massol's results [25] extracted from body-fitted simulations and taken here as reference results, and to the drag force results computed using Lagrange extrapolation in order to assess the Aslam extension effect on the drag force computation.

(a)

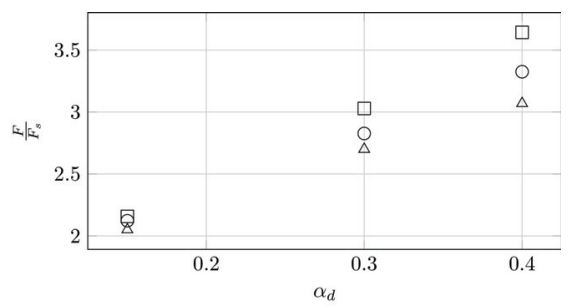

(c)

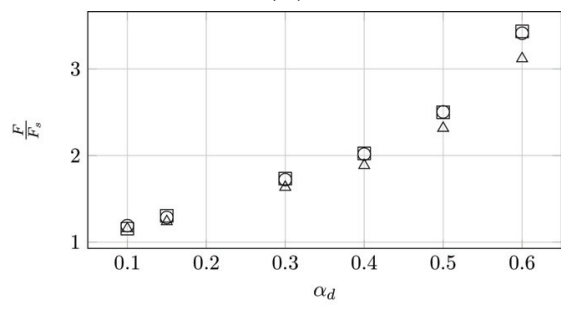

(b)

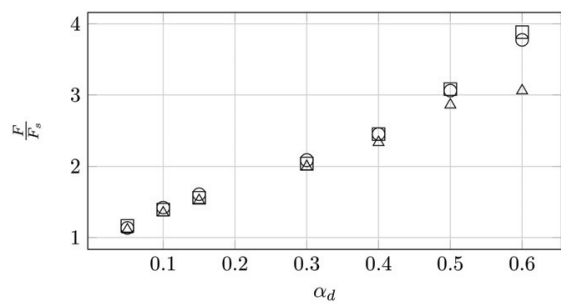

(d)

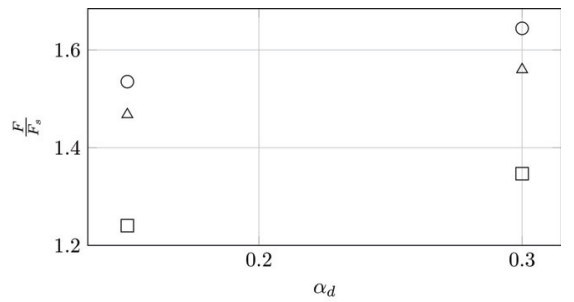

Figure 22. Drag force for a uniform flow past a FCC, normalized by Schiller and Naumann [31] drag force for a uniform flow past an isolated sphere. Results are presented as a function of the solid volume fraction $\alpha_{d}$ and Reynolds number $(a) R e=10,(b) R e=50,(c) R e=100,(d) R e=300:(\square)$ Massol [25], $(\bigcirc)$ present work using Aslam extension and $(\triangle)$ present work using Lagrange extrapolation.

Figure 22 shows the non-dimensional normalized drag force $\frac{F}{F_{s}}$ computed using Aslam extension, the one computed using Lagrange extrapolation and finally the Massol's results [25] used here as a reference to illustrate the possible improvement brought by the Aslam extension applied to the drag force computation. One can observe that for $R e=10,50,100$ the drag force computed using Aslam extension converge toward the reference body fitted values provided by Massol [25]. Aslam simulations match Massol results better than the values computed using Lagrange extrapolation. This shows clearly the positive effect of the reduction, by Aslam extension, of the extrapolation distance $\delta$ on the drag force computation. It is worth noting that Massol simulations were under resolved for $R e=300$ which could explain the discrepancies with our results.

\section{Nusselt coefficient}

Although Aslam extension improvement was observed for an isolated sphere on the Nusselt number results (unlike for the drag force), a study of its effect is conducted here for a FCC array of spheres in order to test its efficiency for the heat transfer computation in an assembly of spheres. The global Nusselt 
coefficient for a uniform flow past a FCC array of spheres is deduced from the Nusselt computed on each sphere by :

$$
\langle N u\rangle=\frac{1}{4} \sum_{i}^{N_{p}} N u_{i}
$$

where $N u_{i}$ is the Nusselt number computed over the $i^{t h}$ particle.

(a)

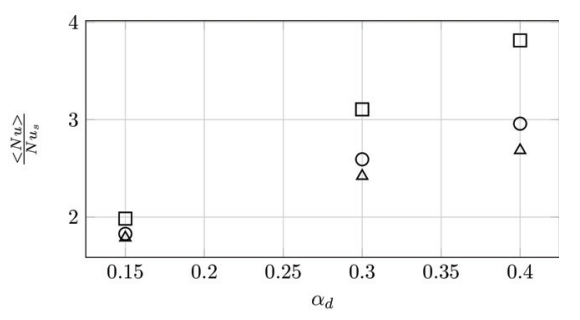

(b)

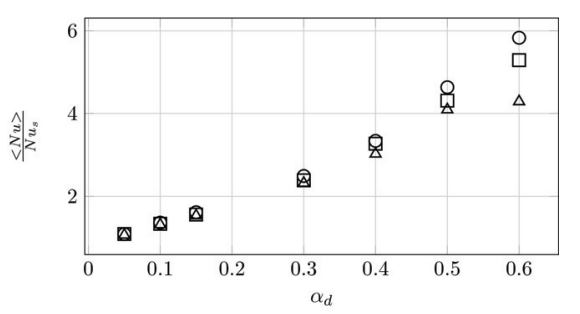

(c)

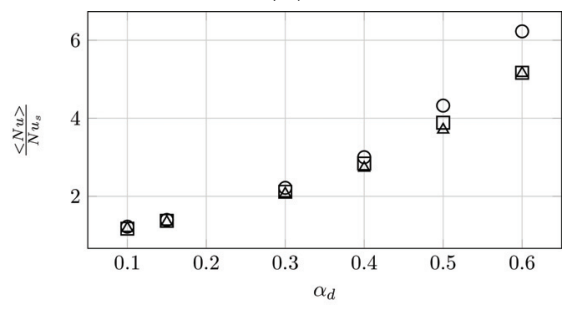

Figure 23. Global Nusselt coefficient for a uniform flow past a FCC, normalized by Ranz and Marshall [29] Nusselt coefficient for a uniform flow past an isolated sphere. Evolution as a function of the solid volume fraction $\alpha_{d}$ at Reynolds numbers $(a) R e=10,(b) R e=50,(c) R e=100$ : ( $)$ ) Massol [25], (○) present work using Aslam extension and $(\triangle)$ present work using Lagrange extrapolation.

Figure 23 shows the normalized Global Nusselt coefficient $\frac{N u}{N u_{s}}$ for a uniform flow past a FCC array of spheres, computed using on one hand Aslam extension and on the other hand Lagrange extrapolation. It also shows $\frac{N u}{N u_{s}}$ extracted from Massol's body fitted simulations [25]. One can observe that Aslam extension improves the heat transfer computation for a packed spheres even if it seems to overestimate it with respect to Massol's results for high solid volume fraction.

\section{Bidisperse Face-Centred Cubic periodic arrangement of spheres}

The last case investigated in this work to assess the possible improvement brought when Aslam extension is used for the drag force computation is a bidisperse Face-Centred Cubic periodic arrangement for two species of spheres : the larger particles are distributed in the same configuration as the one previously presented for monodisperse arrangements while the smaller particles are located at the center of the vertices and at the center of the cubic simulation domain as illustrated in figure 24 . The parameter to set up this case were fully detailed in [7].

The two species are characterized by the following dimensionless parameters :

$$
x_{i}=\frac{\alpha_{i}}{\alpha_{d}} \quad, \quad y_{i}=\frac{d_{i}}{d_{s}}
$$

where $d_{i}$ and $\alpha_{i}$ are the particle diameter and the solid volume fraction of the specie $i$ respectively. The Sauter mean diameter $d_{s}$ is given by :

$$
d_{s}=\left[\sum_{i}^{2} \frac{x_{i}}{d_{i}}\right]^{-1}
$$




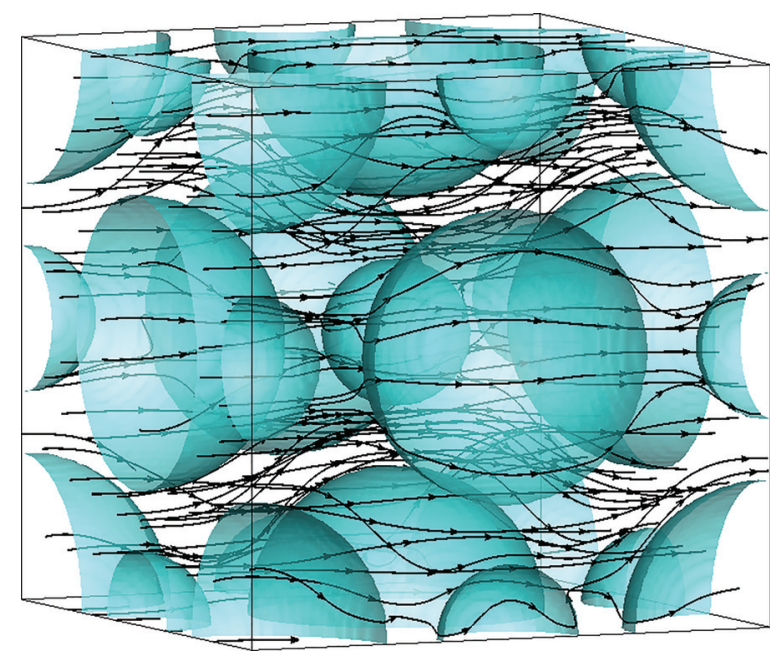

Figure 24. FCC/FCC bidisperse arrangement of spheres for $R e=50$ and $\alpha_{d}=0.3$ - the particle shape is plotted in blue and the black lines are the streamlines.

(a)

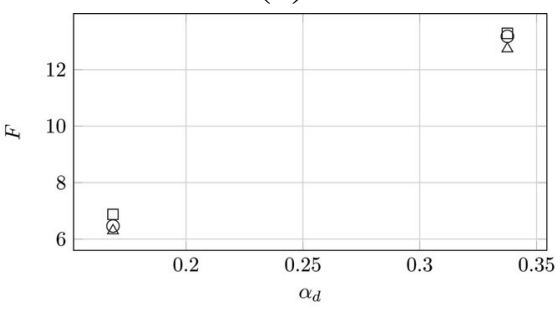

(c)

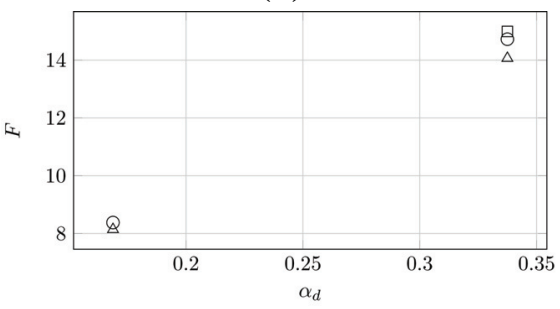

(b)

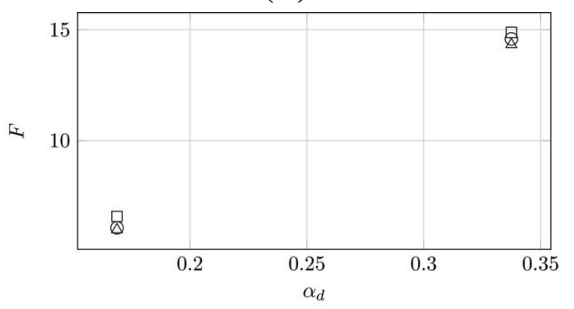

(d)

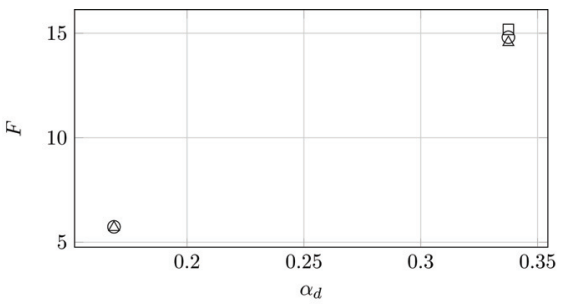

Figure 25. Non-dimensional drag force $F$ for a uniform flow past a FCC/FCC packed spheres as function of the solid volume fraction $\alpha_{d}(a):(R e=50, y 1),(b):(R e=50, y 2),(c):(R e=100, y 1)$, $(d):(R e=100, y 2),:(\square)$ Massol [25], , (○) present work using Aslam extension and $(\triangle)$ present work using Lagrange extrapolation.

Figure 25 shows the non-dimensional drag force $F$ for a uniform flow past a FCC/FCC packed spheres computed using Aslam extension as well as the one computed using Lagrange extrapolation [7]. The results are compared to the body fitted simulations of Massol in terms of drag force, taken as a reference values. It can be observed that drag forces computed using Aslam extension are closer to Massol's results than those computed using Lagrange extrapolation, and that for both species $y_{1}$ and $y_{2}$ at $R e=50,100$.

\section{Conclusions}

An original method has been designed for estimating forces and heat transfer in Particle-resolved Direct Numerical Simulations. It is based on Aslam extension coupled with Taylor interpolation both of third order. This method was first validated without flow resolution for various particle shapes (spherical, 
ellipsoidal and cubical particles) and a numerical study has been conducted in both two- and threedimensions. The expected convergence orders were obtained for spherical and ellipsoidal particles. This method is constructed to be a better alternative to the approach proposed for drag force and heat transfer estimates published in [7] and [6] respectively. This improvement was shown by comparing the results of both techniques applied to multiple test cases. First, in a uniform flow past an isolated hot sphere, Aslam extension use reduces significantly the extrapolation distance. It also correct some inaccurate local pressure values on the sphere. The Aslam extension effect on the drag and Nusselt coefficients was more significant in more complex flows as mono- and bi-disperse Faced-Centered Cubic arrangement of spheres.

The general conclusion that we have obtained is that drag force and heat transfer calculation using Aslam extensions fits to body fitted simulations of Massol et al. [25] better than the computation using Lagrange extrapolations.

Future works will be devoted to applying our hydrodynamic force and heat transfer calculation method to random arrangements of spherical and ellipsoidal particles and extracting correlation laws for these configurations.

\section{Acknowledgements}

This work was granted access to the HPC resources of CINES under the allocation A0032b06115 made by GENCI (Grand Equipement National de Calcul Intensif), and to the resources of CALMIP supercomputing center under the allocation 2017-P1529."

\section{Bibliographie}

J. Adanez, A. Abad, F. Garcia-Labiano, P. Gayan, and L. F. de Diego. Progress in Chemical-Looping Combustion and Reforming technologies. Progress in Energy and Combustion Science, 38(2) :215-282, Apr. 2012.

H. Ali and S. Rohani. Dynamic modeling and simulation of a riser-type fluid catalytic cracking unit. Chemical Engineering and Science, 20 :118-130, 1997.

T. D. Aslam. A partial differential equation approach to multidimensional extrapolation. Journal of Computational Physics, 193(1) :349-355, Jan. 2004.

G. Bagheri, C. Bonadonna, I. Manzella, and P. Vonlanthen. On the characterization of size and shape of irregular particles. Powder Technology, 270 :141-153, Jan. 2015.

R. Beetstra, M. A. van der Hoef, and J. A. M. Kuipers. Drag force of intermediate Reynolds number flow past mono- and bidisperse arrays of spheres. AIChE Journal, 53(2) :489-501, Feb. 2007.

M.-A. Chadil, S. Vincent, and J.-L. Estivalezes. Accurate calculation of heat transfer coefficients for motions around particles with a finite-size particle approach. In $5^{\text {th }}$ Turbulence and Interactions TI 2018 conference, to be submitted in Notes in Numerical Fluid Mechanics and Multidisciplinary Design, Springer, Les Trois-Ilets, French West Indies, France, 2018.

M.-A. Chadil, S. Vincent, and J.-L. Estivalezes. Accurate estimate of drag forces usingparticle-resolved direct numerical simulations. under revision in Acta Mechanica, 2018.

M.-A. Chadil, S. Vincent, and J.-L. Estivalezes. Drag, lift and Nusselt coefficients for ellipsoid using Particle-Resolved Direct Numerical Simulations. In $5^{\text {th }}$ Turbulence and Interactions TI 2018 conference, to be submitted in Notes in Numerical Fluid Mechanics and Multidisciplinary Design, Springer, Les Trois-Ilets, French West Indies, France, 2018.

M.-A. Chadil, S. Vincent, and J.-L. Estivalezes. Improvement of the viscous penalty method for particle-resolved simulations. Submitted in Open Journal of Fluid Dynamics, 2018. 
B. P. L. Clair, A. E. Hamielec, and H. R. Pruppacher. A numerical study of the drag on a sphere at low and intermediate reynolds numbers. Journal of the Atmospheric Sciences, 27(2) :308-315, 1970.

S. Dartevelle. From model conception to verification and validation, a global approach to multiphase Navier-Stoke models with an emphasis on volcanic explosive phenomenology. Technical Report LA-14346, 948564, Oct. 2007.

N. G. Deen, S. H. Kriebitzsch, M. A. van der Hoef, and J. Kuipers. Direct numerical simulation of flow and heat transfer in dense fluid-particle systems. Chemical Engineering Science, 81 :329-344, Oct. 2012.

S. C. R. Dennis and J. D. A. Walker. Calculation of the steady flow past a sphere at low and moderate reynolds numbers. Journal of Fluid Mechanics, 48(4) :771-789, 1971.

S. Ergun. Fluid flow through packed columns. Chemical Engineering Progress, (48) :89-94, 1952.

Z.-G. Feng and E. E. Michaelides. A numerical study on the transient heat transfer from a sphere at high Reynolds and Peclet numbers. International Journal of Heat and Mass Transfer, 43(2) :219-229, 2000.

A. Gobin, H. Neau, O. Simonin, J.-R. Llinas, V. Reiling, and J.-L. Sélo. Fluid dynamic numerical simulation of a gas phase polymerization reactor. International Journal for Numerical Methods in Fluids, 43(10-11) :1199-1220, 2003.

D. Gunn. Transfer of heat or mass to particles in fixed and fluidized beds. International Journal of Heat and Mass Transfer, 21(4) : $467-476,1978$.

R. J. Hill, D. L. Koch, and A. J. C. Ladd. Moderate-Reynolds-number flows in ordered and random arrays of spheres. Journal of Fluid Mechanics, 448, Dec. 2001.

M. A. V. D. Hoef, R. Beetstra, and J. A. M. Kuipers. Lattice-Boltzmann simulations of low-Reynolds-number flow past mono- and bidisperse arrays of spheres : results for the permeability and drag force. Journal of Fluid Mechanics, 528, Apr. 2005.

D. Lacanette, S. Vincent, A. Sarthou, P. Malaurent, and J.-P. Caltagirone. An eulerian/lagrangian method for the numerical simulation of incompressible convection flows interacting with complex obstacles: Application to the natural convection in the lascaux cave. International Journal of Heat and Mass Transfer, 52 :2528-2542, 2009.

A. J. C. Ladd. Numerical simulations of particulate suspensions via a discretized Boltzmann equation. Part 1. Theoretical foundation. Journal of Fluid Mechanics, 271 :285, July 1994.

A. J. C. Ladd. Numerical simulations of particulate suspensions via a discretized Boltzmann equation. Part 2. Numerical results. Journal of Fluid Mechanics, 271 :311, July 1994.

A. Lyngfelt, B. Leckner, and T. Mattisson. A fuidized-bed combustion process with inherent CO2 separation; application of chemical-looping combustion. Chemical Engineering Science, page 13, 2001.

J. Magnaudet, M. Rivero, and J. Fabre. Accelerated flows past a rigid sphere or a spherical bubble. Part 1. Steady straining flow. Journal of Fluid Mechanics, 284(-1) :97, Feb. 1995.

A. Massol. Simulations numériques d'écoulements à travers des réseaux fixes de spheres monodisperses et bidisperses, pour des nombres de Reynolds modérés. PhD thesis, 2004.

T. Mattisson, M. Keller, C. Linderholm, P. Moldenhauer, M. Rydan, H. Leion, and A. Lyngfelt. Chemical-looping technologies using circulating fluidized bed systems : Status of development. Fuel Processing Technology, 172 :1-12, Apr. 2018 .

A. Ozel, J. B. de Motta, M. Abbas, P. Fede, O. Masbernat, S. Vincent, J.-L. Estivalezes, and O. Simonin. Particle resolved direct numerical simulation of a liquid-solid fluidized bed : Comparison with experimental data. International Journal of Multiphase Flow, 89 :228-240, 2017.

T. Randrianarivelo, G. Pianet, S. Vincent, and J.-P. Caltagirone. Numerical modelling of the solid particle motion using a new penalty method. International Journal for Numerical Methods in Fluids, 47 :1245-1251, 2005.

W. E. Ranz and W. R. Marshall. Evaporation from drops. Parts I \& II. Chem. Eng. Progr., pages 48 :141-6; 173-80, 1952.

A. Sarthou, S. Vincent, P. Angot, J.-P. Caltagirone, and others. The sub-mesh penalty method. Finite Volumes for Complex Applications V, 2009.

L. Schiller and A. Z. Naumann. Über die grundlegenden Berechnungen bei der Schwerkraftaufbereitung. Ver. Deut. Ing., $77: 318-320,1933$.

T. Schönfeld and M. Rudgyard. Steady and Unsteady Flow Simulations Using the Hybrid Flow Solver AVBP. AIAA Journal, 37(11) :1378-1385, Nov. 1999.

B. Sun, S. Tenneti, and S. Subramaniam. Modeling average gas-solid heat transfer using particle-resolved direct numerical simulation. International Journal of Heat and Mass Transfer, 86 :898-913, July 2015. 
P. K. Sweby. High Resolution Schemes Using Flux Limiters for Hyperbolic Conservation Laws. SIAM Journal on Numerical Analysis, 21(5):995-1011, Oct. 1984.

H. Tavassoli, S. Kriebitzsch, M. van der Hoef, E. Peters, and J. Kuipers. Direct numerical simulation of particulate flow with heat transfer. International Journal of Multiphase Flow, 57 :29-37, Dec. 2013.

S. Tenneti, R. Garg, and S. Subramaniam. Drag law for monodisperse gas-solid systems using particle-resolved direct numerical simulation of flow past fixed assemblies of spheres. International Journal of Multiphase Flow, 37(9):10721092, Nov. 2011.

S. Tenneti, B. Sun, R. Garg, and S. Subramaniam. Role of fluid heating in dense gas-solid flow as revealed by particleresolved direct numerical simulation. International Journal of Heat and Mass Transfer, 58(1-2) :471-479, Mar. 2013.

M. Uhlmann. An immersed boundary method with direct forcing for the simulation of particulate flows. Journal of Computational Physics, 209(2) :448-476, 2005.

M. Uhlmann. Interface-resolved direct numerical simulation of vertical particulate channel flow in the turbulent regime. Physics of Fluids, 20(5):053305, 2008.

S. Vincent, J. C. B. de Motta, A. Sarthou, J.-L. Estivalezes, O. Simonin, and E. Climent. A Lagrangian VOF tensorial penalty method for the DNS of resolved particle-laden flows. Journal of Computational Physics, 256 :582-614, 2014.

Z. Wang, J. Fan, and K. Luo. Combined multi-direct forcing and immersed boundary method for simulating flows with moving particles. International Journal of Multiphase Flow, 34(3) :283 - 302, 2008.

C.-Y. Wen and Y. H. Yu. Mechanics of fluidization. Chemical Engineering Progress Symposium Series, (62) :100-111, 1966.

S. Whitaker. Forced convection heat transfer correlations for flow in pipes, past flat plates, single cylinders, single spheres, and for flow in packed beds and tube bundles. AIChE Journal, 18(2) :361-371, 1972.

M. Zastawny, G. Mallouppas, F. Zhao, and B. van Wachem. Derivation of drag and lift force and torque coefficients for non-spherical particles in flows. International journal of Multiphase Flow, (39) :227-239, 2012. 